\title{
REAVALIANDO A RELAÇÃO ENTRE PRODUTIVIDADE E EXPORTAÇÕES ATRAVÉS DE MODELO DE CORREÇÃO DE ERROS NÃO LINEAR: EVIDÊNCIAS PARA ESTADOS UNIDOS, CANADÁ, JAPÃO E ALEMANHA
}

\author{
João Paulo Martin Faleiros* \\ Denisard Cneio de Oliveira Alves ${ }^{\dagger}$
}

\begin{abstract}
Resumo
O presente artigo avalia a relação entre exportações e produtividade total dos fatores (PFT) através do modelo de correção de erros não linear MR-STVEC (Multiple Regime Smooth Transition VEC) aplicado a quatro países desenvolvidos (Estados Unidos, Canadá, Japão e Alemanha). O MRSTVEC, assim como os demais da família de modelos de transição suave, exige uma estratégia prévia de identificação. Tomando como base a estratégia de Lundbergh et al. (2003), são aplicados dois diferentes testes de razão de Máxima Verossimilhança. Os resultados indicam que, para todos os casos, as exportações possuem mecanismo de reverter possíveis choques negativos de produtividade. Adicionalmente, para o Canadá e Alemanha, quando há choques positivos da produtividade, as exportações também agem, mas de modo a restringi-los.
\end{abstract}

Palavras-chave: Exportações; Produtividade; Modelos não lineares.

\begin{abstract}
The aim of this paper is to evaluate the relationship between exports and productivity using Multiple Regimes Smooth Transition Vector ErrorCorrection Model (MR-STVEC) for a sample of four developed countries (United States, Canada, Japan and Germany). MR-STVEC models require a previous strategy of identification. Based on the strategy used by Lundbergh et al. (2003), we apply two different likelihood ratio tests. The results indicate that exports may reverse negative productivity shocks. In particular, for Canada and Germany, exports are able to act as a restriction to an increase in productivity when there are positive shocks.
\end{abstract}

Keywords: Exports; Productivity; Nonlinear models.

JEL classification: C22, C51, F43.

DOI: http://dx.doi.org/10.1590/1413-8050/ea 120727

\footnotetext{
* Banco Nacional de Desenvolvimento Econômico e Social (BNDES) Brasil. E-mail: jpfaleiros@hotmail.com

$\dagger$ Faculdade de Economia e Administração da USP (FEA-USP).E-mail: dcoalves@usp.br
} 


\section{Introdução}

Em geral, a literatura aponta que, para alguns países, essas séries cointegram e a produtividade é destaque na literatura econômica (Krueger 1980, Bruton 1989, Edwards 1993, 1998, Bernard \& Jensen 1999, Vamvakidis 2002). Entretanto, o que ainda é motivo de fortes controvérsias é a direção de causalidade pela qual essa relação vem a ocorrer.

Por um lado está a corrente econômica tradicional que advoga que a expansão das exportações orienta a produtividade de um país (Balassa 1978, Feder 1982, Esfahani 1991, Marin 1992, Rodrik 1995, De Loecker 2007). De maneira geral, a expansão das exportações pode ser um canal pelo qual a nação pode auferir ganhos de competitividade e lucratividade. Esse fato viria a ocorrer por meio da melhoria na alocação de recursos do país, de acordo com sua vantagem comparativa, tal como pela exploração de economias de escala. Outro benefício ligado à expansão das exportações é a difusão de conhecimento através de "spillovers" tecnológicos gerado pela forte competição no mercado mundial. Quando as firmas têm contato com o mercado internacional, passam a adquirir conhecimento e expertise técnica que permitem a melhoria de seu nível de eficiência e produtividade, ou seja, um mecanismo de learning-by-exporting.

De outro lado, estão aqueles que questionam esse mecanismo de propagação. Segundo eles, não existe necessariamente dinâmica de crescimento que se processa das exportações para a produtividade. Pelo contrário, a expansão das exportações seria reposta ao aumento da produtividade doméstica (Krugman 1984, Bhagwati 1998, Grossman \& Helpman 1991, Edwards 1993, Coe \& Helpman 1995). Recentemente alguns estudos no âmbito da firma vêm contestando a dinâmica de learning-by-exporting como fonte de impacto sobre o crescimento, trazendo evidências de que somente as firmas mais produtivas se lançam às exportações, influenciando diretamente sua performance (Aw et al. 1997, Clerides et al. 1998, Bernard \& Jensen 1999, Bernard et al. 2007).

Para avaliar o sentido de causalidade entre exportações e produtividade, ou mesmo outra medida de desempenho econômico, boa parte da literatura empírica faz uso da abordagem de séries de tempo, principalmente dos modelos de correção de erros (ECM), associado ao teste de cointegração de Johansen $(1988,1991)$, metodologia presentes nos principais trabalhos empíricos sobre o tema (Marin 1992, Henriques \& Sadorsky 1996, Xu 1996, Yamada 1988, Awokuse 2007). Em geral, a literatura aponta que, para alguns países, essas séries cointegram e a produtividade é a variável responsável pelo ajuste de curto prazo (Greenaway \& Sapsford 1994, Giles \& Williams 2000a,b). Portanto, choques positivos nas exportações irão se traduzir em crescimento da produtividade, corroborando a visão tradicional.

Em contrapartida, esse resultado traz consigo outro aspecto associado ao padrão de causalidade. De acordo com o mecanismo padrão de correção de erros (ECM), quando há incidência de choques positivos de produtividade, este é dissipado, dado que as exportações não ajustam. Em especial para nações com baixa participação do setor exportador na economia, este não parece ser comportamento crível. Nesse caso, espera-se o contrário, ou seja, que as exportações passem a ser endógenas, de modo que choques de produtividade tenham reflexos sobre o setor exportador. Consequentemente, a variável responsável pelo ajustamento de curto prazo alternaria de acordo com o sinal dos choques de produtividade, indicando um mecanismo não linear de correção 
de erros.

Este artigo traz evidência de que, primeiramente, existe relação de longo prazo entre a produtividade total dos fatores e receitas de exportações, avaliadas em termos de sua participação no valor total exportado pelo mundo, para quatro países de alta renda per capita e níveis diferentes de participação das exportações no PIB: Estados Unidos, Canadá, Japão e Alemanha. O resultado mais importante é que a dinâmica de ajustamento de curto prazo, dada pelo vetor de correção de erros, é não linear e especificada de acordo com o modelo MR-STVEC (Multiple Regime Smooth Transition VEC), que justamente permite alternância de regimes em função dos choques de produtividade.

Além de corroborar a visão tradicional, de que choques nas exportações promovem aumento da produtividade, será também evidenciado que nem sempre essa última variável é fracamente endógena. Considerando o caso japonês e alemão, choques positivos de produtividade promovem aumento da razão entre a participação das receitas de exportações e o valor total exportado pelo mundo. Já no caso de choques negativos, todos os quatro modelos MR-STVEC estimados indicam que a produtividade continua sendo a variável responsável pelo ajuste, implicando movimento de reversão desta. Por fim, mesmo levando em conta a especificação não linear, os modelos para Canadá e Alemanha trouxeram evidências de dinâmica de restrição da produtividade em reposta a choques positivos.

Vale destacar que a estimação dos modelos MR-STVEC, assim como toda a família desses modelos, exige estratégia de especificação, que neste artigo irá se basear no trabalho de Lundbergh et al. (2003). Como aqui o tratamento é bivariado, serão aplicados dois testes de razão de verossimilhança (LR).

$\mathrm{O}$ artigo é organizado em mais cinco seções. A seção 2 trará mais considerações a respeito da relação entre produtividade total dos fatores e exportações. A seção 3 apresenta o modelo MR-STVEC, seguida da seção 4 que abordará a estratégia de especificação. Por sua vez, a seção 5 discutirá o modo como as séries foram construídas e também os resultados obtidos. Finalmente, a seção 6 conclui este trabalho.

\section{Considerações sobre a relação entre produtividade e exportaões}

Como destacado, a literatura econômica, de modo geral, não possui muitas restrições de ordem teórica para relacionar exportações à produtividade de um país. Tal como salienta Kunst \& Marin (1989) e Marin (1992), até mesmo por meio de breve exame empírico, é possível verificar trajetória conjunta entre exportações e produtividade, incluindo nessa análise, não só países em desenvolvimento, mas aqueles com elevado nível de renda per capita. De fato, ao realizar inspeção casual entre as séries da produtividade total dos fatores $\left(P T F_{t}\right)$ de quatro países nessa faixa de renda, no caso, Estados Unidos, Canadá, Alemanha e Japão, e a participação das receitas de exportações de bens no valor total exportado mundialmente $\left(E X_{t}\right)$, nota-se que as essas duas séries coevoluem ao longo dos últimos 60 anos, como aponta a Figura 1.

A produtividade total dos fatores foi calculada como resíduo de uma típica função Cobb-Douglas, tal que o produto seja função do estoque de capital e do número total de trabalhadores. ${ }^{1}$ Vale destacar que a participação das exporta-

\footnotetext{
${ }^{1}$ Como propõe Hall \& Jones (1999), assumiu-se que a elasticidade do capital é igual a 1/3, e
} 

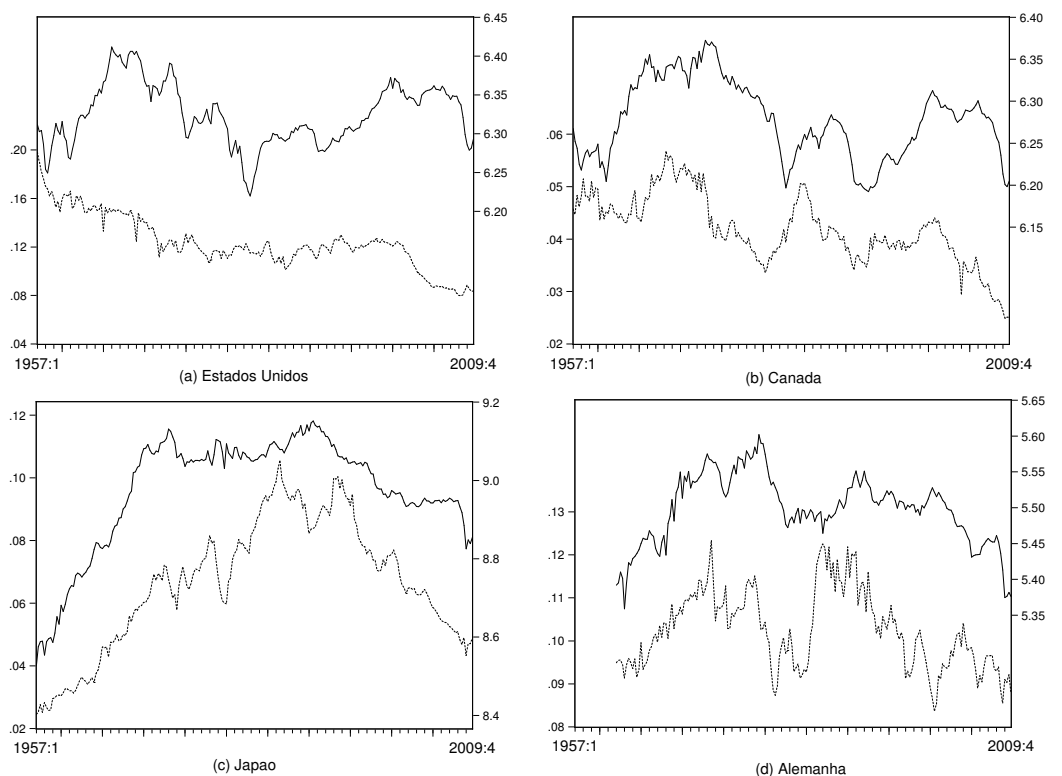

Fonte: Elaboração dos autores.

Figura 1: Evolução trimestral do $\log$ da $P T F_{t}$ (linha cheia) e do índice $E X_{t}$ (linha tracejada)

ções de bens do país no total do valor exportado pelo mundo (EX), além de ser uma boa medida para caracterizar a trajetória de expansão das exportações, pode também ser considerada proxy para a produtividade do setor exportador. ${ }^{2}$ Isso vem a significar, por exemplo, que aumentos da participação das exportações de um país no total exportado pelo mundo indicam a existência de alguma contrapartida favorável na produtividade do setor exportador, independentemente do sentido de orientação de causalidade em que isso venha a acontecer. ${ }^{3}$

No contexto aqui proposto, se houver indício de cointegração e de que a dinâmica de ajustamento se processa pela PTF, conclui-se que o movimento de causalidade de longo prazo se processa das exportações para a produtividade total dos fatores. Contudo, o vetor de correção de erros estimado irá impli-

com base no trabalho de Issler et al. (2004), foi considerado que o progresso tecnológico do país evolui exogenamente, de acordo com o modelo de Solow padrão. O estoque de capital inicial foi calculado tomando como base o procedimento de Hall \& Jones (1999), considerando que o consumo de capital fixo foi utilizado como o montante depreciado a cada trimestre. Os dados foram obtidos do Fundo Monetário Internacional. Mais detalhes sobre o cálculo da PTF serão destacados adiante na seção 5 deste artigo.

${ }^{2}$ Essa suposição torna-se factível, uma vez que esse indicador, de modo bem abrangente, acaba por refletir os mecanismos econômicos convencionalmente propostos pela literatura que associa o papel das exportações das nações à sua produtividade. Pode-se citar a ocorrência de exploração de economias de escala, a melhora relativa da eficiência técnica, a melhoria dos termos de troca, dentre outros.

${ }^{3}$ A ideia principal para utilização desse índice é de justamente captar os movimentos antagônicos das exportações do país em relação ao total exportado pelo mundo. Por exemplo, quando há uma contrapartida favorável no índice, mesmo que seja via denominador, vem a significar que as exportações do país ainda se mantêm competitiva no comércio internacional. O país pode não ter sido influenciado pela tendência mundial de queda das exportações. 
car, nesse caso, que a produtividade total dos fatores, já descontada dos efeitos do indicador de competitividade das exportações (PTFD), é determinada como resíduo da relação de longo prazo. Portanto, sua evolução torna-se meramente reativa à dinâmica de ajustamento ao equilíbrio deste sistema. Dada a conjunção de vários fatores que atuam sobre a PTF, essa restrição torna-se pouco factível, principalmente para países com setor não exportador amplo.

Via de regra, a literatura que faz o uso dessa abordagem empírica não leva em conta esse aspecto, pois grande parte das análises que testam a hipótese de orientação de crescimento via exportações se concentra em países em desenvolvimento que apresentam, em geral, economias com mercado doméstico pequeno e matriz produtiva preponderantemente voltada para fora. ${ }^{4}$ Entretanto, quando se analisa países como os Estados Unidos e Japão, como propõe o artigo de Marin (1992), em que há mercado interno relativamente amplo e forte presença de setores com bens não comercializáveis, o modelo linear torna-se pouco factível.

Portanto, o que se faz necessário é relaxar a suposição imposta pelo vetor de correção de erros convencional de que, necessariamente, as exportações sempre restringem potenciais choques positivos de produtividade, não relacionados com o padrão de competitividade da atividade exportadora, fazendo com que a PTFD seja puramente reativa. Sendo assim, o que se sugere neste artigo é a hipótese de que essa dinâmica de ajustamento é não linear, tal que haja alternância entre regimes definidos tanto pelos desvios ao equilíbrio de longo prazo, como pelos regimes de retorno, que pode se processar de dois modos: via indicador de competitividade das exportações ou pela própria PTF.

Quais os motivos de ordem teórica para a existência desse padrão não linear? Para responder a essa questão, assume-se que a economia é divida em dois principais setores. O primeiro agrega as firmas ligadas ao setor exportador, todavia, não exime a possibilidade de atuação no mercado doméstico. Já o segundo setor agrega as firmas não exportadoras com produção orientada exclusivamente ao mercado doméstico. Além disso, nessa economia, há possibilidade de spillovers tecnológicos entre os setores, possibilitando a existência de uma relação de longo prazo entre as produtividades setoriais e, portanto, a relação de longo prazo entre a PTF da economia e a produtividade do setor exportador. Como a análise aqui é dinâmica, assume-se adicionalmente que haveria processo de ajustamento no caso de eventuais desequilíbrios nessa relação. A questão, portanto, é identificar qual produtividade será responsável pelo ajuste, e isso vai estar diretamente relacionado às características de cada economia.

Nesse contexto, suponha uma economia com razão produto por trabalhador elevada, em que há mercado doméstico bastante amplo, com predominância da produção no segundo setor e baixa participação do primeiro setor no PIB. A ocorrência de choques positivos de produtividade, oriundos do setor não exportador, por exemplo, promoverá desvio em relação ao equilíbrio inicial. Como consequência, espera-se que o setor exportador seja responsável pelo restabelecimento do equilíbrio, uma vez que o aumento de produtividade do setor voltado ao mercado doméstico promove aumento da PTF que, por conseguinte, gera externalidades positivas à produtividade do setor ex-

\footnotetext{
${ }^{4}$ Em economias menores, torna-se mais provável que a escala de eficiência mínima seja maior do que o tamanho do mercado doméstico, indicando a necessidade de voltar parte de sua produção ao comércio internacional.
} 
portador, elevando sua competitividade no comércio internacional. Ou seja, nesse regime, a produtividade total dos fatores orienta a competitividade das exportações.

Por outro lado, a ocorrência de choques negativos no setor não exportador também promoverá desvio em relação ao equilíbrio inicial, fazendo a PTF decrescer. Como a produtividade do primeiro setor responde à dinâmica competitiva no mercado internacional, há possibilidade desse choque não propiciar declínio em sua produtividade e, consequentemente, no nível de competitividade no comércio internacional. Como tendência de curto prazo, espera-se que o ajustamento se processe pelo próprio setor orientado para o mercado doméstico. O setor exportador, portanto, agiria como sustentador da PTF, revertendo a trajetória de queda. Isso poderia estar relacionado, por exemplo, ao aprofundamento da geração de externalidades positivas do primeiro para o segundo setor, tal como os termos de troca poderiam se tornar mais favoráveis às exportações, ou até mesmo, um deslocamento de firmas que passariam a comercializar seus produtos internacionalmente. Vale destacar que a velocidade de ajustamento aqui evidenciada dependerá de quão promissor é o mecanismo de catch-up das externalidades entre os setores.

Suponha agora uma economia que também possui relação produto por trabalhador elevada, mas com mercado doméstico menor e com boa parte de sua produção orientada para fora, conduzindo-o então à participação elevada das exportações no PIB. O mecanismo de reversão ao choque negativo de produtividade, orientada pelo setor exportador, não seria muito diferente da economia descrita anteriormente. Entretanto, quando há choques positivos de produtividade advindos do setor não exportador, não necessariamente implicará que a produtividade do setor exportador reaja endogenamente como se espera em países com mercado doméstico amplo, uma vez que em economias menores é possível que a produtividade do setor exportador possua menor sensibilidade de resposta à evolução da produtividade do setor orientado domesticamente. Poderia existir, por exemplo, no setor exportador mecanismo de desincentivo de captura de externalidades oriundas do setor não exportador. A sobrevivência de uma inovação, advinda do setor não exportador, é posta em xeque se o setor exportador não apropriá-la, tal como o custo de viabilizá-la pode ser muito superior aos benefícios a serem auferidos no comércio internacional. Portanto, haveria uma dissipação desse choque e o restabelecimento do equilíbrio ocorreria por meio do segundo setor, e, por conseguinte, da própria PTF. Consequentemente, nessa economia, o setor exportador agiria de modo a restringir a elevação da produtividade do país.

Toda a dinâmica de ajustamento, portanto, vai estar ligada ao regime de crescimento em que a PTF e a PTFD se encontram, seja em uma trajetória de crescimento ou de queda. Destacando que quanto maior o mecanismo de catch-up de externalidades entre os setores, maior a velocidade de ajuste ao equilíbrio. A Tabela 1, a seguir, apresenta a dinâmica não linear de desvio e de retorno ao equilíbrio de longo prazo entre a PTF e o indicador de competitividade das exportações, considerada aqui proxy para a produtividade do setor exportador.

Existem quatro diferentes situações em que o ajustamento está ou não habilitado, a depender das características de cada país. Considerando $d>e$, os regimes 2 e 3 refletem, respectivamente, os mecanismos de restrição e sustentação da PTF, caso seja esta a variável responsável pelo ajuste ao equilíbrio. Por sua vez, o regime 1 indica o desvio negativo à trajetória de longo prazo, 
Tabela 1: Mecanismo não linear de desvio e de retorno ao equilíbrio de longo prazo em caso de choques sobre a PTF

\begin{tabular}{|c|c|c|c|c|c|c|}
\hline Regime & $\sum \Delta P T F_{t-d}($ choque $)$ & $\sum \triangle P T F D_{t-e}$ & Movimento & $\begin{array}{l}\text { Ajuste via } \\
\triangle P T F_{t-1}\end{array}$ & $\begin{array}{l}\text { Ajuste via } \\
\triangle E X_{t-1}\end{array}$ & Mecanismos de Ajuste \\
\hline 1 & $<0$ & $<0$ & $\begin{array}{l}\text { Desvio ao Equilíbrio de } \\
\text { Longo Prazo }(<0)\end{array}$ & Não & Não & - \\
\hline \multirow[t]{2}{*}{2} & \multirow[t]{2}{*}{$>0$} & \multirow[t]{2}{*}{$<0$} & \multirow[t]{2}{*}{$\begin{array}{l}\text { Retorno ao Equilíbrio } \\
\text { de Longo Prazo }(<0)\end{array}$} & Não & Sim & $\begin{array}{l}\text { Aumento de EX em reposta à ele- } \\
\text { vação da PTF }\end{array}$ \\
\hline & & & & Sim & Não & EX restringe aumento da PTF \\
\hline 3 & $<0$ & $>0$ & $\begin{array}{l}\text { Retorno ao Equilíbrio } \\
\text { de Longo Prazo }(>0)\end{array}$ & $\begin{array}{l}\text { Sim } \\
\text { Não }\end{array}$ & $\begin{array}{l}\text { Não } \\
\text { Sim }\end{array}$ & $\begin{array}{l}\text { EX reverte queda da PTF } \\
\text { Diminuição de EX em reposta à } \\
\text { queda da PTF }\end{array}$ \\
\hline 4 & $>0$ & $>0$ & $\begin{array}{l}\text { Desvio ao Equilíbrio de } \\
\text { Longo Prazo }(>0)\end{array}$ & Não & Não & - \\
\hline
\end{tabular}


em que há um processo de decrescimento da PTF acumulada sucedido do declínio acumulado da PTFD, enquanto que o regime 4 indica o movimento inverso de perturbação do sistema dinâmico, com ascensão do acumulado da PTF sucedido de trajetória positiva do acumulado da PTFD.

Nessas circunstâncias, a família de modelos econométricos autorregressivos com transição suave é considerada aqui a mais apropriada para caracterizar essa dinâmica, uma vez que possibilita incorporar os quatro tipos regimes de ajustamento ao equilíbrio de longo prazo de acordo com a evolução de $\sum \triangle P T F_{t-d}$ e $\sum \triangle P T F D_{t-e}$, descritos na Tabela 1.

\section{O modelo MR-STVEC}

Seguindo a proposta de Dijik et al. (2002) para modelos multivariados da família STAR, o modelo MR-STVEC com $p$ defasagens e quatro regimes é especicado como:

$$
\begin{aligned}
\Delta x_{t}= & \left(\mu_{1}+\alpha_{1} z_{t-1}+\sum_{j=1}^{p} \Phi_{1, j} \Delta x_{t-j}\right)\left(1-F\left(s_{F_{t-d}}\right)\right)\left[1-G\left(s_{G_{t-e}}\right)\right]+ \\
& \left(\mu_{2}+\alpha_{2} z_{t-1}+\sum_{j=1}^{p} \Phi_{2, j} \Delta x_{t-j}\right) F\left(s_{F_{t-d}}\right)\left[1-G\left(s_{G_{t-e}}\right)\right]+ \\
& \left(\mu_{3}+\alpha_{3} z_{t-1}+\sum_{j=1}^{p} \Phi_{3, j} \Delta x_{t-j}\right)\left(1-F\left(s_{F_{t-d}}\right)\right) G\left(s_{G_{t-e}}\right)+ \\
& \left(\mu_{4}+\alpha_{4} z_{t-1}+\sum_{j=1}^{p} \Phi_{4, j} \Delta x_{t-j}\right) F\left(s_{F_{t-d}}\right) G\left(s_{G_{t-e}}\right)+\varepsilon_{t}
\end{aligned}
$$

tal que, $x_{t}=\left(x_{1 t}, x_{2 t}, \ldots, x_{k t}\right)^{\prime}$ é um vetor $(k \times 1)$ séries de tempo; $\Delta$ é o operador da primeira diferença; $\mu_{i}$ para $i=1,2,3,4$ são vetores $(k \times 1)$ de constantes; $\alpha_{i}$ são matrizes $(k \times r)$ de coeficientes de ajustamento, tal que $r$ é o número de vetores de cointegração; $z_{t}=\beta^{\prime} x_{t}$, tal que, $\beta$ é matriz $(k \times r)$ das relações lineares de longo prazo; $\Phi_{i, j}$ para $i=1,2,3,4$ e $j=1, \ldots, p$ são matrizes dos coeficientes das defasagens; e $\varepsilon_{t}$ é o vetor de ruídos brancos com média 0 e variância $\Sigma$. As funções $F\left(s_{F_{t-d}}\right)$ e $G\left(s_{G_{t-e}}\right)$ são logísticas ${ }^{5}$ e limitadas entre 0 e 1 , de acordo com Anderson \& Teräsvirta (1992), Teräsvirta (1994) e Dijik et al. (2002), portanto,

$$
\begin{aligned}
& F\left(s_{F_{t-d}}\right)=\left(1+\exp \left(\frac{-\gamma_{F}\left(s_{F_{t-d}}-c_{F}\right)}{\hat{\sigma}_{s_{F}}}\right)\right)^{-1} \\
& G\left(s_{G_{t-e}}\right)=\left(1+\exp \left(\frac{-\gamma_{G}\left(s_{G_{t-e}}-c_{G}\right.}{\hat{\sigma}_{s_{G}}}\right)\right)^{-1}
\end{aligned}
$$

tal que, $s_{F_{t}}$ e $s_{G_{t}}$ são as variáveis de transição, $\gamma>0$ é o parâmetro de suavidade (smooth parameter); $d$ e $e$ são os parâmetros de atraso (delay parameters) das funções de transição, $\hat{\sigma}_{s_{F}}$ e $\hat{\sigma}_{s_{G}}$ são os desvios padrões de $s_{F_{t}}$ e $s_{G_{t}}$, respectivamente; $c_{F}$ e $c_{G}$ são os respectivos limiares (thresholds) de cada função

\footnotetext{
${ }^{5}$ Optou-se por utilizar a função logística uma vez que possibilita alternância entre dois tipos de regimes com características distintas, diferentemente da função exponencial que considera os valores extremos da série como único regime. Portanto, os desvios ao regime normal de crescimento representam apenas um regime.
} 
de transição e indicam o momento em que ocorre a mudança de regime. ${ }^{6}$ Portanto, o modelo possui relação de longo prazo linear, mas dinâmica de retorno ao equilíbrio não linear. ${ }^{7}$ Assume-se, adicionalmente, que o modelo é globalmente estacionário, mas que pode apresentar regimes com trajetória de forte persistência. Logo, para avaliar se de fato as séries podem ser descritas de acordo com o modelo de múltiplos regimes, é necessário realizar inicialmente testes de especificação para a estrutura multivariada com duas funções logísticas.

\section{Especificação do Modelo MR-STVEC}

A necessidade de uma estratégia coerente de especicação é requerida para avaliar se as séries podem ser caracterizadas adequadamente pelo modelo (1). Considera-se nesse processo, a escolha das variáveis de transição e a identicação dos parâmetros de atraso $d$ ou $e$. Caso não haja indícios de que o melhor modelo é aquele descrito por (1), avalia-se se as séries podem ser caracterizadas por vetor de correção de erros com apenas uma função de transição (STVEC), como aplicado em Rothman et al. (2001), ou mesmo um VEC convencional. Nesse sentido, dois procedimentos serão executados para a decisão do modelo mais apropriado para sistema com duas equações.

O primeiro procedimento recomendado por Granger \& Teräsvirta (1993), recorrentemente aplicado na literatura dos modelos da família STAR, é denominado especíco para o geral. Consiste em realizar testes de especicação, a começar pela estimação do modelo de vetor de correção de erros mais simples, no caso o VEC, passando pelo STVEC, até o mais complexo, o MR-STVEC. O segundo procedimento, denominado especíco para o geral para o especíco, foi proposto em Lundbergh et al. (2003) e consiste em avaliar diretamente se o modelo MR-STVEC é mais apropriado do que o VEC e a partir daí é avaliado se a especicação STVEC com diferentes variáveis de transição é isoladamente apropriada para caracterizar as séries em questão.

Para a análise e melhor entendimento dos procedimentos aqui adotados, convém reprarametrizar o modelo descrito em (1), da seguinte maneira:

$$
\begin{aligned}
\Delta x_{t}=\tilde{\mu}_{1}+\tilde{\alpha}_{1} z_{t-1}+\sum_{j=1}^{p} \tilde{\Phi}_{1, j} \Delta x_{t-j}+\left(\tilde{\mu}_{2}+\tilde{\alpha}_{2} z_{t-1}+\sum_{j=1}^{p} \tilde{\Phi}_{2, j} \Delta x_{t-j}\right) F^{*}\left(s_{F_{t-d}}\right)+ \\
\\
\left(\tilde{\mu}_{3}+\tilde{\alpha}_{3} z_{t-1}+\sum_{j=1}^{p} \tilde{\Phi}_{3, j} \Delta x_{t-j}\right) G^{*}\left(s_{G_{t-e}}\right)+ \\
\left(\tilde{\mu}_{4}+\tilde{\alpha}_{4} z_{t-1}+\sum_{j=1}^{p} \tilde{\Phi}_{4, j} \Delta x_{t-j}\right) F^{*}\left(s_{F_{t-d}}\right) G^{*}\left(s_{G_{t-e}}\right)+\varepsilon_{t}
\end{aligned}
$$

tal que, $\tilde{\mu}_{1}=\mu_{1}, \tilde{\mu}_{2}=\mu_{2}-\mu_{1}, \tilde{\mu}_{3}=\mu_{3}-\mu_{1}, \tilde{\mu}_{4}=\mu_{1}+\mu_{4}-\mu_{3}-\mu_{2}, \tilde{\alpha}_{1}=\alpha_{1}, \tilde{\alpha}_{2}=$ $\alpha_{2}-\alpha_{1}, \tilde{\alpha}_{3}=\alpha_{3}-\alpha_{1}, \tilde{\alpha}_{4}=\alpha_{1}+\alpha_{4}-\alpha_{3}-\alpha_{2}, \tilde{\Phi}_{1, j}=\Phi_{1, j}, \tilde{\Phi}_{2, j}=\Phi_{2, j}-\Phi_{1, j}, \tilde{\Phi}_{3, j}=$ $\Phi_{3, j}-\Phi_{1, j}$ e $\tilde{\Phi}_{4, j}=\Phi_{1, j}+\Phi_{4, j}-\Phi_{3, j}-\Phi_{2, j}$. Além do mais, $F^{*}\left(s_{F_{t-d}}\right)=F\left(s_{F_{t-d}}\right)-$ $\frac{1}{2}$ e $G^{*}\left(s_{G_{t-e}}\right)=G\left(s_{G_{t-e}}\right)-\frac{1}{2}$. A reparametrização em (4) facilita enunciar as

\footnotetext{
${ }^{6}$ Se $\gamma \rightarrow \infty$, a função de transição possui transição discreta entre os regimes tal como a família dos modelos TAR (Threshold Autorregressive).

${ }^{7}$ O MR-STVEC pode ser considerado uma extensão do modelo de Rothman et al. (2001). Com essa estrutura, nos extremos 0 e 1 das funções de transição, o modelo assume 4 diferentes tipos de regimes.
} 
hipóteses nulas de linearidade $H_{0}: \gamma_{F}=\gamma_{G}=0, H_{0}: \gamma_{F}=0$ e $H_{0}: \gamma_{G}=0$ a serem testadas em cada procedimento. Entretanto, sob as hipóteses nulas enunciadas o MR-STVEC possui parâmetros não identificáveis. A literatura, neste caso, recomenda a realização da expansão de Taylor de primeira ordem ao redor de $\gamma_{F}=\gamma_{G}=0$. Sendo assim, (4) pode ainda ser descrita como:

$$
\begin{aligned}
\Delta x_{t}= & \mu_{1}^{*}+\alpha_{1}^{*} z_{t-1}+\sum_{j=1}^{p} \Phi_{1, j}^{*} \Delta x_{t-j}+\left(\mu_{2}^{*}+\alpha_{2}^{*} z_{t-1}+\sum_{j=1}^{p} \Phi_{2, j}^{*} \Delta x_{t-j}\right) s_{F_{t-d}}+ \\
& \left(\mu_{3}^{*}+\alpha_{3}^{*} z_{t-1}+\sum_{j=1}^{p} \Phi_{3, j}^{*} \Delta x_{t-j}\right) s_{G_{t-e}}+ \\
& \left(\mu_{4}^{*}+\alpha_{4}^{*} z_{t-1}+\sum_{j=1}^{p} \Phi_{4, j}^{*} \Delta x_{t-j}\right) s_{F_{t-d}} G_{t-e}+R\left(\gamma_{F}, \gamma_{G}\right)+\varepsilon_{t}
\end{aligned}
$$

Os coeficientes a serem estimados $\mu_{i}^{*}, \alpha_{i}^{*}$ e $\Phi_{i, j}^{*}$ para $i=1,2,3,4$ e $j=$ $1, \ldots, p$ são combinações lineares dos coeficientes $\tilde{\mu}_{i}, \tilde{\alpha}_{i}$ e $\tilde{\Phi}_{i, j}$ em (4). O termo $R\left(\gamma_{F}, \gamma_{G}\right)$ representa o resto da expansão de Taylor de primeira ordem. Sob a hipótese nula esse termo é igual a zero, não trazendo prejuízo à distribuição do teste. A partir de (5) é possível executar os dois procedimento aqui indicados para avaliar se a especificação em (1) é adequada.

\subsection{Procedimento "Específico para o Geral"}

Inicia-se esse procedimento com o teste de linearidade proposto em Teräsvirta (1994), adaptado para sistema de duas equações, onde se avalia a hipótese nula de linearidade $H_{0}: \gamma_{F}=0$ contra a hipótese alternativa $H_{0}: \gamma_{F}>0$. Tomando como base a especificação (4), assumindo-se a princípio que $\tilde{\mu}_{3}=\tilde{\mu}_{4}=$ $0, \tilde{\alpha}_{3}=\tilde{\alpha}_{4}=0$ e $\tilde{\Phi}_{3, j}=\tilde{\Phi}_{4, j}=0$, é avaliado se o modelo com apenas uma função de transição $F\left(s_{F_{t-d}}, \gamma_{F}, c_{F}\right)$ é o mais apropriado do que o modelo linear. Como este modelo possui parâmetros não identificáveis sob a hipótese nula $H_{0}: \gamma_{F}=0$, realiza-se expansão de Taylor de primeira ordem ao redor de $\gamma_{F}=0$. O resultado dessa expansão pode ser descrita por (5), assumindo-se que $\mu_{i}^{*}=\alpha_{i}^{*}=\Phi_{i, j}^{*}=0$, para $i=3,4$. O teste de razão de verossimilhança (LR) é apropriado uma vez que se trata de sistema com mais de uma equação.

Refutada a hipótese nula de linearidade, estima-se modelo com uma função de transição $\left(S T V E C_{F}\right)$ por mínimos quadrados não lineares. Em seguida, executa-se novo teste LR tomando como base o "remaining nonlinearity test" proposto por Eitrheim \& Teräsvirta (1996) e discutido em Dijik et al. (2002), modificado para a estrutura de sistema de mais de uma equação. Refutada a hipótese de não linearidade adicional, há evidências de que as séries podem ser caracterizadas por (1).

As etapas detalhadas para a implementação dos testes são apresentadas a seguir.

A. Estimar o modelo VEC linear e reportar os resíduos de cada uma das equações.

B. Regredir os resíduos do passo anterior sobre a constante, $z_{t-1}$ e $\Delta x_{t-j}$ para $j=1, \ldots, p$ e computar a matriz de variância-covariância $\Sigma_{11}$. 
C. Regredir os resíduos do VEC linear sobre a constante, $z_{t-1}, z_{t-1} s_{F_{t-d}}$ e $\Delta x_{t-j} s_{F_{t-d}}$ para $j=1, \ldots, p$ e computar a matriz de variância-covariância $\Sigma_{12}$.

D. Calcular a estatística $L R_{1}=(T-c)\left\{\log \left(\Sigma_{11}\right)-\log \left(\Sigma_{12}\right)\right\}$ distribuída de acordo com uma $\chi^{2}$ com grau de liberdade igual ao número de restrições do sistema, em que $T$ é o número de observações usadas e $c$ (correção de Sims para pequenas amostras) é igual ao número de parâmetros estimados em cada equação do sistema não restrito.

E. Caso se refute a hipótese nula de linearidade, a pelo menos $10 \%$ de significância, estima-se o sistema de equações por mínimos quadrados não lineares multivariado. Como em Teräsvirta (1994) e Lundbergh et al. (2003), o valor do parâmetro de atraso $d$ e da variável de transição $s_{F_{t-d}}$ são escolhidos com base no menor p-valor do teste de linearidade. ${ }^{8}$

Define-se o modelo $S T V E C_{F}$ estimado através da seguinte especificação:

$$
\begin{aligned}
\Delta x_{t} & =H\left(z_{t-1}, \Delta x_{t-j}, s_{F_{t-d}} ; \hat{\Psi}\right)+\hat{\varepsilon}_{t} \\
& =H\left(z_{t-1}, \Delta x_{t-j}, s_{F_{t-d}} ; \hat{\tilde{\mu}}_{1}, \hat{\tilde{\mu}}_{2}, \hat{\tilde{\alpha}}_{1}, \hat{\tilde{\alpha}}_{2}, \hat{\tilde{\Phi}}_{1, j}, \hat{\tilde{\Phi}}_{2, j}, \hat{\gamma}_{F}, \hat{c}_{F}\right)+\hat{\varepsilon}_{t}
\end{aligned}
$$

tal que,

$$
\begin{aligned}
H\left(z_{t-1}, \Delta x_{t-j}, s_{F_{t-d}} ; \hat{\Psi}\right)= & \hat{\tilde{\mu}}_{1}+\hat{\tilde{\alpha}}_{1} z_{t-1}+\sum_{j=1}^{p} \hat{\tilde{\Phi}}_{1, j} \Delta x_{t-j}+\hat{\varepsilon}_{t}+ \\
& \left(\hat{\tilde{\mu}}_{2}+\hat{\tilde{\alpha}}_{2} z_{t-1}+\sum_{j=1}^{p} \hat{\tilde{\Phi}}_{2, j} \Delta x_{t-j}\right) F\left(s_{F_{t-d}}, \hat{\gamma}_{F}, \hat{c}_{F}\right)
\end{aligned}
$$

F. Regredir o resíduo do modelo $S T V E C_{F}$ sobre $\nabla H\left(z_{t-1}, \Delta x_{t-j}, s_{F_{t-d}} ; \hat{\Psi}\right)=$ $\frac{\partial H\left(z_{t-1}, \Delta x_{t-j}, s_{F_{t-d}} ; \hat{\Psi}\right)}{\partial \hat{\Psi}},{ }^{9}$ para assim obter a matriz de variância-covariância do modelo restrito $\Sigma_{21}$.

G. Regredir cada resíduo do modelo $S T V E C_{F}$ sobre $\nabla H\left(z_{t-1}, \Delta x_{t-j}, s_{F_{t-d}} ; \hat{\Psi}\right)$ $=\frac{\partial H\left(z_{t-1}, \Delta x_{t-j}, s_{F_{t-d}} ; \hat{\Psi}\right)}{\partial \hat{\Psi}}$ e sobre a expansão de Taylor de primeira ordem da função $G\left(s_{G_{t-e}}, \gamma_{G}, c_{G}\right)$, em torno de $\gamma_{G}=0$, ou seja, $z_{t-1} s_{G_{t-e}}, \Delta x_{t-j} s_{G_{t-e}}$, $z_{t-1} F\left(s_{F_{t-d}}, \hat{\gamma}_{F}, \hat{c}_{F}\right) s_{G_{t-e}}$ e $\Delta x_{t-j} F\left(s_{F_{t-d}}, \hat{\gamma}_{F}, \hat{c}_{F}\right) s_{G_{t-e}}$ para $j=1, \ldots, p$ e obter a matriz de variância-covariância do modelo não restrito $\Sigma_{22} \cdot{ }^{10}$

H. Calcular a estatística $L R_{2}=(T-c)\left\{\log \left(\sum_{21}\right)-\log \left(\sum_{22}\right)\right\}$ distribuída de acordo com uma $\chi^{2}$ com grau de liberdade igual ao número de restrições do sistema, em que $T$ é o número de observações usadas e $c$ (correção de Sims para pequenas amostras) é igual ao número de parâmetros estimados em cada equação do sistema não restrito. Utiliza-se novamente

\footnotetext{
${ }^{8}$ Ver detalhes em Teräsvirta (1994, p. 211) e Lundbergh et al. (2003, p. 107 e 113).

${ }^{9}$ Para mais detalhes sobre a derivada parcial com relação aos coeficientes estimados do STVEC, consultar Apêndice A.

${ }^{10} \mathrm{O}$ artigo de Dijik \& Franses (1999) apresenta procedimento semelhante para o caso univariado.
} 
como critério o menor p-valor para a escolha da variável de transição $s_{G_{t-e}}$ e do parâmetro de atraso $e$.

Se a hipótese nula é refutada, então o MR-STVEC pode ser estimado por mínimos quadrados não lineares. Encerra-se, portanto, o procedimento "específico para o geral".

\subsection{Procedimento "Específico para o Geral para o Específico"}

Neste procedimento, Lundbergh et al. (2003) propõem testar diretamente a especicação linear contra a especicação do MR-STVEC. Se a hipótese nula de linearidade for rejeitada, testam-se duas sub-hipóteses em que se avalia se os modelos $S T V E C_{F}$ e $S T V E C_{G}$ são individualmente necessários para a caracterização das séries. Se essas duas hipóteses forem refutadas, temos indícios de que as séries podem ser descritas como um MR-STVEC. Os testes são realizados por meio da estimação do modelo (5). Os passos necessários para aplicar o procedimento especíco para o geral para o especíco são apresentados a seguir:

A. Estimar o modelo VEC linear e reportar os resíduos de cada uma das equações.

B. Regredir o vetor de resíduos do passo anterior sobre $z_{t-1}$ e $\Delta x_{t-j}$ para $j=1, \ldots, p$ e computar a matriz de variância-covariância $\tilde{\Sigma}_{11}$.

C. Regredir o vetor de resíduos do VEC linear sobre $z_{t-1}, \Delta x_{t-j}, z_{t-1} s_{F_{t-d}}$, $\Delta x_{t-j} s_{F_{t-d}}, z_{t-1} s_{G_{t-e}}, \Delta x_{t-j} s_{G_{t-e}}, z_{t-1} s_{F_{t-d}} s_{G_{t-e}}$ e $\Delta x_{t-j} s_{F_{t-d}} s_{G_{t-e}}$ para $j=1, \ldots, p$ e computar $\tilde{\Sigma}_{12}$.

D. Calcular a estatística $L R_{1}^{\prime}=(T-c)\left\{\log \left(\tilde{\Sigma}_{11}\right)-\log \left(\tilde{\Sigma}_{12}\right)\right\}$ que é distribuída de acordo com uma $\chi^{2}$ com grau de liberdade igual ao número de restrições do sistema, em que $T$ é o número de observações usadas e $c$ (correção de Sims para pequenas amostras) é igual ao número de parâmetros estimados em cada equação do sistema não restrito.

E. Se rejeitarmos a hipótese nula $H_{0}^{M R-S T V E C}$ de que o modelo é um VEC, a pelo menos $10 \%$ de significância, realiza-se dois testes de hipótese auxiliares adicionais $H_{0}^{S T V E C_{F}}$ e $H_{0}^{S T V E C_{G}}$ que avaliam se os modelos $S T V E C_{F}$ e $S T V E C_{G}$ de modo isolado são bons para caracterizar as séries de tempo. Ou seja, regredir, respectivamente, o vetor de resíduos estimados do VEC linear sobre $z_{t-1}, \Delta x_{t-j}, z_{t-1} s_{G_{t-e}}$ e $\Delta x_{t-j} s_{G_{t-e}}$ para $j=1, \ldots, p$ e computar a matriz de variância e covariância $\tilde{\Sigma}_{13}$, assim como, regredir o resíduos estimados obtidos do modelo VEC linear sobre $z_{t-1}, \Delta x_{t-j}, z_{t-1} s_{F_{t-d}}$ e $\Delta x_{t-j} s_{F_{t-d}}$ para $j=1, \ldots, p$ e computar a matriz de variância e covariância $\tilde{\Sigma}_{14}$.

F. Calcular as estatísticas $L R_{3}^{\prime}=(T-c)\left\{\log \left(\tilde{\Sigma}_{13}\right)-\log \left(\tilde{\Sigma}_{12}\right)\right\}$ e $L R_{4}^{\prime}=(T-$ c) $\left\{\log \left(\tilde{\Sigma}_{14}\right)-\log \left(\tilde{\Sigma}_{12}\right)\right\}$ distribuídas de acordo com $\chi^{2}$ uma com grau de liberdade igual ao número de restrições do sistema, em que $T$ é o número de observações usadas e $c$ (correção de Sims para pequenas amostras) é igual ao número de parâmetros estimados em cada equação do sistema não restrito. 
Após a execução dos passos apresentados, se a hipótese nula $H_{0}^{M R-S T V E C}$ é rejeitada, assim como $H_{0}^{S T V E C_{F}}$ e $H_{0}^{S T V E C_{G}}$ são rejeitadas, há indícios de que individualmente os modelos $S T V E C_{F}$ e $S T V E C_{G}$ são necessários para caracterizar as séries de tempo, portanto, estima-se o $M R-S T V E C$. No caso de a hipótese nula $H_{0}^{S T V E C_{F}}$ ser rejeitada, mas $H_{0}^{S T V E C_{G}}$ não ser rejeitada, significa que individualmente o $S T V E C_{G}$ não é apto para caracterização, portanto, somente o modelo $S T V E C_{F}$ deve ser estimado. O mesmo raciocínio é válido quando $H_{0}^{S T V E C_{G}}$ for rejeitada e $H_{0}^{S T V E C_{F}}$ não for rejeitada. ${ }^{11}$

\section{Avaliação dos Resultados}

\subsection{Dados}

Para este estudo foram obtidas as séries trimestrais e com ajuste sazonal do PIB, formação bruta de capital, total da força de trabalho e receita de exportações de bens em dólares, a preços correntes, para os Estados Unidos, Canadá, Japão e Alemanha, além da série do valor total exportado de bens no mundo em dólares, a preços correntes. As séries foram obtidas do banco de dados International Financial Statistic do FMI e abrangem o período entre o primeiro trimestre de 1957 ao quarto trimestre de 2009, exceção feita ao caso alemão, em que as séries iniciam-se no primeiro trimestre de 1962. Somente para as séries de exportações de bens foi necessário realizar o ajuste sazonal. ${ }^{12}$

A série $\left\{E X_{t}\right\}$ foi calculada por meio da razão entre as receitas de exportações nominais do país e o total de receitas do exportado pelo mundo. Para o computo da produtividade total dos fatores $\left\{P T F_{t}\right\}$, as séries do PIB e da formação bruta de capital foram deflacionadas para o ano de 2005, por meio do deflator do PIB de cada país, também obtido da base de dados do FMI. O estoque de capital foi estimado com base na proposta de Hall \& Jones (1999). O montante inicial do estoque de capital foi obtido pelo cálculo da razão entre o valor inicial da série de formação bruta de capital $\left(I_{0}\right)$ e a soma entre a taxa geométrica de crescimento trimestral da formação bruta de capital nos 10 primeiros anos da série $g$ e a taxa média de depreciação $\delta$, portanto, $\frac{I_{0}}{(g+\delta)}$. A base de dados do FMI também fornece o consumo de capital fixo, que após ser deflacionada, foi utilizada como montante depreciado em cada trimestre, tal como para a estimativa da taxa média de depreciação $\delta$. Por simplicidade, assumiu-se que o valor da elasticidade do capital é igual a 1/3 (Cooley \& Prescott 1995, Hall \& Jones 1999). Em vez de se assumir valor fixo para o coeficiente do progresso tecnológico exógeno $\theta$, optou-se por estimá-lo através de mínimos quadrados.

As variáveis de transição para o modelo MR-STVEC foram construídas através do cálculo do somatório da variação trimestral da produtividade total dos fatores $\left(\triangle \log P T F_{t}\right)$, variando de 1 a 8 trimestres $\left(h_{1}=1, \ldots, 8\right)$, e da produtividade total dos fatores descontada $\left(\triangle P T F D_{t}\right)$, também variando de 1 a 8 trimestres $\left(h_{2}=1, \ldots, 8\right)$, ou seja,

\footnotetext{
${ }^{11} \mathrm{O}$ mecanismo não linear de ajustamento aqui proposto ainda não foi discutido pela literatura. Portanto, torna-se necessário avaliar o poder e tamanho do teste para ambos os procedimentos, levando-se em conta os possíveis mecanismos de correção de erros presente nesta análise. Tal exercício esta presente no Anexo C deste artigo disponível somente na versão online.

${ }^{12} \mathrm{O}$ ajuste sazonal foi realizado com base no método Census X12.
} 


$$
\begin{array}{lll}
s_{F_{t-d}}=\left(\sum_{h=1}^{h=h_{1}} \Delta \log P T F_{t-h+1}\right)_{t-d} & \text { ou } & \left(\sum_{h=1}^{h=h_{2}} \Delta \log P T F D_{t-h+1}\right)_{t-d} \\
s_{G_{t-e}}=\left(\sum_{h=1}^{h=h_{1}} \Delta \log P T F_{t-h+1}\right)_{t-e} & \text { ou } & \left(\sum_{h=1}^{h=h_{2}} \Delta \log P T F D_{t-h+1}\right)_{t-e}
\end{array}
$$

Vale destacar que a $P T F D_{t}$ é calculada como resíduo da relação de longo prazo entre o log da $P T F_{t}$ e a razão das exportações $E X_{t}$.

\subsection{Resultados dos Testes de Especificação}

Os testes de especificação propostos foram realizados com base na estrutura do VEC linear estimado pelo método de Johansen $(1988,1991)$. O critério de informação de Akaike (AIC) foi utilizado para a escolha do número de defasagens do sistema, levando-se em conta também a ausência de autocorrelação nos resíduos de cada equação. ${ }^{13}$ Para todos os casos, o teste traço de razão de verossimilhança indicou a existência de um vetor de cointegração, como aponta a Tabela B.1 no Apêndice B.

Os parâmetros de atraso (8) e (9) são dados por $d=1, \ldots, d_{\text {max }}$ e $e=1, \ldots, e_{\text {max }}$, com $d_{\text {max }}=e_{\max }=4$. Uma vez que o procedimento "específico para o geral para o específico" envolve a realização do teste para o total de 1024 diferentes modelos estimados, a Tabela 2, apresenta somente os cinco menores p-valores ligados à hipótese $H_{0}^{M R-S T V E C}$ e os subtestes auxiliares para cada país aqui em análise.

Ainda em relação aos resultados da Tabela 2, considerou-se que o valor do parâmetro de atraso $d$ é necessariamente superior ao parâmetro $e$. Lembrando que a escolha dos parâmetros de atraso e do valor acumulado das taxas de crescimento das variáveis de transição segue a proposta de Teräsvirta (1994), ou seja, selecionar os parâmetros que apresentarem o menor p-valor. Para todos os países, há indícios de que a hipótese nula $H_{0}^{M R-S T V E C}$ é rejeitada a pelo menos $1 \%$ de significância. Do mesmo modo, as duas sub-hipóteses $H_{0}^{S T V E C_{F}}$ e $H_{0}^{S T V E C_{G}}$ foram rejeitadas a níveis convencionais de significância. Logo, de acordo com o procedimento em questão, há fortes evidências de que as séries podem ser descritas de acordo com um MR-STVEC.

Resta verificar se o procedimento "específico para o geral" também conduz às mesmas conclusões. Repare que em grande parte dos casos, $h_{1}$ e $h_{2}$ apresentaram valores superiores a 1 , conferindo a variável de transição valores acumulados da taxa de crescimento trimestral das séries que podem chegar próximos a 2 anos, como no caso alemão.

A coluna 4 da Tabela 3 apresenta os p-valores do teste do procedimento "específico para o geral", cuja hipótese nula verifica se a especificação do modelo $S T V E C_{F}$ é mais adequada do que o modelo VEC linear. Concomitantemente, a coluna 8 apresenta os p-valores do teste em que se verifica se a especificação do modelo $S T V E C_{G}$ é mais adequada do que o modelo VEC linear.

O procedimento "específico para o geral" apresenta resultados compatíveis ao procedimento anterior. Observa-se que para todos os pares $\left(h_{1}, d\right)$ destacados na Tabela 3, houve rejeição da hipótese nula quando associada a $F($.$) , a pelo menos 5 \%$ de significância, com p-valores inferiores aos pares $\left(h_{2}\right.$, e) correspondentes. Seguindo a estratégia de Lundbergh et al. (2003), faz-se

\footnotetext{
${ }^{13}$ Os resultados do teste Dickey-Fuller de raiz unitária, não reportados, indicaram que todos os processos $\log P T F_{t}$ e de $E X_{t}$ são integrados de ordem 1.
} 
Tabela 2: p-valores do Teste L para o procedimento "específico para o geral para o específico"

\begin{tabular}{|c|c|c|c|c|c|c|c|}
\hline \multirow{3}{*}{ Países } & \multirow{2}{*}{\multicolumn{2}{|c|}{$\begin{array}{l}\text { Variável de Transição F(.) } \\
\left(\sum_{h=1}^{h=h_{1}} \Delta \log P T F_{t-h+1}\right)_{t-d}\end{array}$}} & \multirow{2}{*}{\multicolumn{2}{|c|}{$\frac{\text { Variável de Transição G(.) }}{\left(\sum_{h=1}^{h=h_{2}} \Delta \log P T F D_{t-h+1}\right)_{t-e}}$}} & \multicolumn{3}{|c|}{ Hipóteses Nulas } \\
\hline & & & & & \multirow[t]{2}{*}{$H_{0}^{M R-S V T E C}$} & \multirow[t]{2}{*}{$H_{0}^{S T V E C_{F}}$} & \multirow[t]{2}{*}{$H_{0}^{S T V E C_{C}}$} \\
\hline & $h_{1}$ & $\mathrm{~d}$ & $h_{2}$ & $\mathrm{e}$ & & & \\
\hline \multirow{5}{*}{ Estados Unidos } & 2 & 4 & 2 & 1 & 0,000006 & 0,000002 & 0,000144 \\
\hline & 2 & 4 & 6 & 3 & 0,000008 & 0,000003 & 0,000178 \\
\hline & 2 & 4 & 7 & 2 & 0,000010 & 0,000001 & 0,000222 \\
\hline & 3 & 4 & 2 & 1 & 0,000012 & 0,000003 & 0,000054 \\
\hline & 5 & 4 & 5 & 1 & 0,000015 & 0,000044 & 0,000033 \\
\hline \multirow{5}{*}{ Canadá } & 3 & 3 & 1 & 2 & 0,000426 & 0,000172 & 0,002604 \\
\hline & 2 & 4 & 1 & 2 & 0,000518 & 0,000216 & 0,006803 \\
\hline & 2 & 4 & 2 & 1 & 0,000754 & 0,000208 & 0,009859 \\
\hline & 3 & 3 & 6 & 2 & 0,000806 & 0,000425 & 0,002758 \\
\hline & 3 & 3 & 7 & 1 & 0,001099 & 0,000139 & 0,003846 \\
\hline \multirow{5}{*}{ Japão } & 1 & 4 & 4 & 3 & 0,000001 & 0,000001 & 0,000121 \\
\hline & 1 & 4 & 3 & 4 & 0,000003 & 0,000001 & 0,000299 \\
\hline & 1 & 4 & 6 & 1 & 0,000033 & 0,000009 & 0,002669 \\
\hline & 3 & 2 & 6 & 1 & 0,000040 & 0,000011 & 0,000394 \\
\hline & 4 & 3 & 1 & 1 & 0,000041 & 0,000089 & 0,000006 \\
\hline \multirow{5}{*}{ Alemanha } & 2 & 2 & 7 & 1 & 0,000011 & 0,000000 & 0,002097 \\
\hline & 2 & 2 & 1 & 1 & 0,000078 & 0,001003 & 0,035171 \\
\hline & 2 & 2 & 2 & 1 & 0,000091 & 0,000018 & 0,039845 \\
\hline & 2 & 2 & 8 & 1 & 0,000163 & 0,000005 & 0,015078 \\
\hline & 2 & 2 & 5 & 1 & 0,000851 & 0,000046 & 0,185063 \\
\hline
\end{tabular}




\begin{tabular}{|c|c|c|c|c|c|c|c|c|}
\hline \multirow{3}{*}{ Países } & \multirow{2}{*}{\multicolumn{2}{|c|}{$\begin{array}{l}\text { Variável de Transição } \mathrm{F}(.) \\
\left(\sum_{h=1}^{h=h_{1}} \log \triangle P T F_{t-h+1}\right)_{t-d}\end{array}$}} & \multicolumn{2}{|c|}{ Hipóteses Nulas associadas a F(.) } & \multicolumn{2}{|c|}{ Variável de Transição G(.) } & \multicolumn{2}{|c|}{ Hipóteses Nulas associadas a G(.) } \\
\hline & & & \multirow[t]{2}{*}{$H_{0}^{V E C}$} & \multirow[t]{2}{*}{$H_{0}^{S T V E C_{F}}$} & \multicolumn{2}{|c|}{$\left(\sum_{h=1}^{h=h_{2}} \log \triangle P T F D_{t-h+1}\right)_{t-e}$} & \multirow[t]{2}{*}{$H_{0}^{V E C}$} & \multirow[t]{2}{*}{$H_{0}^{S T V E C_{G}}$} \\
\hline & $h_{1}$ & $\mathrm{~d}$ & & & $h_{2}$ & $\mathrm{e}$ & & \\
\hline \multirow[b]{3}{*}{ Estados Unidos } & 2 & 4 & 0,00143 & 0,05830 & 2 & 1 & 0,10190 & 0,01771 \\
\hline & 2 & 4 & 0,00143 & 0,05830 & 6 & 3 & 0,11249 & 0,02606 \\
\hline & 2 & 4 & 0,00143 & 0,05830 & 7 & 2 & 0,27539 & 0,07192 \\
\hline \multirow{5}{*}{ Canadá } & 3 & 3 & 0,01057 & 0,20571 & 1 & 2 & 0,14581 & 0,04792 \\
\hline & 2 & 4 & 0,00378 & 0,41775 & 1 & 2 & 0,14581 & 0,06495 \\
\hline & 2 & 4 & 0,00378 & 0,41775 & 2 & 1 & 0,15705 & 0,01220 \\
\hline & 3 & 3 & 0,01057 & 0,20571 & 6 & 2 & 0,15668 & 0,43239 \\
\hline & 3 & 3 & 0,01057 & 0,20571 & 7 & 1 & 0,45580 & 0,43221 \\
\hline Japão & 1 & 4 & 0,00023 & 0,00428 & 4 & 3 & 0,04406 & 0,00244 \\
\hline \multirow{5}{*}{ Alemanha } & 2 & 2 & 0,00001 & 0,15404 & 7 & 1 & 0,71918 & 0,01463 \\
\hline & 2 & 2 & 0,00001 & 0,15404 & 1 & 1 & 0,00328 & 0,16036 \\
\hline & 2 & 2 & 0,00001 & 0,15404 & 2 & 1 & 0,23594 & 0,10768 \\
\hline & 2 & 2 & 0,00001 & 0,15404 & 8 & 1 & 0,67799 & 0,07020 \\
\hline & 2 & 2 & 0,00001 & 0,15404 & 5 & 1 & 0,65335 & 0,51445 \\
\hline
\end{tabular}


necessário estimar os modelos STVEC com a função de transição $F($.$) e nova-$ mente realizar os testes de especificação, só que dessa vez, para avaliar se o modelo MR-STVEC é mais adequado do que o modelo STVEC. Os p-valores do segundo estágio dos testes para as funções de transição $F($.$) e G($.$) já es-$ timados são apresentados, respectivamente, nas colunas 5 e 9 da Tabela 3. Vale ainda destacar que, de maneira similar aos resultados das simulações por Monte Carlo, no Apêndice $C$, os testes $L R$ sob as hipóteses nulas associadas a $G($.) apresentaram níveis de rejeição maiores quando comparado ao estágio anterior.

Por conseguinte, verifica-se que há evidências suficientes, com base na estratégia de especificação aqui delineada de que as séries do log da PTF e da razão das exportações podem ser descritas de acordo com um MR-STVEC. No caso alemão, não houve dificuldades para a escolha dos parâmetros. Os pares $\left(h_{1}=2, d=2\right)$ e $\left(h_{2}=7, e=1\right)$ propiciaram os menores p-valores no "procedimento específico para o geral para o específico" tal como nas duas hipóteses nulas dos estágios do procedimento "específico para o geral". O mesmo se verifica para o Japão com os pares $\left(h_{1}=1, d=4\right)$ e $\left(h_{2}=4, e=3\right)$. Já no caso canadense, devido ao melhor ajuste do modelo, observado pelo critério de informação $A I C$, selecionou-se as combinações $\left(h_{1}=2, d=4\right)$ e $\left(h_{2}=2, e=1\right)$, que são compatíveis com os menores p-valores em cada estágio do procedimento "específico para o geral". Somente para os Estados Unidos, optou-se por escolher os pares $\left(h_{1}=3, d=4\right)$ e $\left(h_{2}=2, e=1\right)$, mesmo que não tenham sido as combinações com os menores p-valores para ambos os procedimentos, uma vez que os modelos MR-STVECs estimados para o par $\left(h_{1}=2, d=4\right)$ não apresentaram o melhor ajustamento aos dados, principalmente pela presença de heterocedasticidade condicional nos resíduos.

Portanto, a partir dos resultados da estratégia de especificação aqui proposta, recorre-se a estimação dos modelos VEC de múltiplos regimes com transição suave.

\subsection{Análise dos Resultados}

Além disso, o teste ARCH aplicado aos resíduos, ou seja, minimiza-se a expressão $\sum_{t} u_{t} \sum u_{t}$, tal que, $u_{t}$ é o vetor de resíduos e $\Sigma=E u_{t}^{\prime} u_{t}$. Um grid com os valores fixos dos coeficientes do parâmetro de suavidade é utilizado para escolha dos valores iniciais. Aqueles que engendrarem o menor log do determinante de $\Sigma$ são utilizados para tanto. Como a proposta empírica discutida na seção 2 implica que a mudança de regimes ocorre de acordo com a alternância dos sinais da taxa de crescimento do acumulado do $\log$ da $P T F_{t}$ e da $P T F D_{t}$, assumiu-se que os valores iniciais dos limiares são iguais a zero.

Os resultados dos modelos estimados para os quatro países em análise são apresentados na Tabela $4 .{ }^{14}$ Alguns pontos são importantes de serem ressaltados previamente à discussão dos resultados. Primeiramente, observa-se que os modelos não lineares se ajustam melhor às séries em destaque, como apontam os critérios de informação $A I C$ e da razão entre os desvios padrões do modelo linear e não linear inferiores à unidade. Além disso, o teste $A R C H$, aplicado aos resíduos de cada equação, não traz indícios da existência de heterocedasticidade condicional, a pelo menos 5\% de significância. Em segundo

\footnotetext{
${ }^{14}$ Para salvar espaço, os coeficientes das defasagens do MR-STVEC não foram reportados.
} 
lugar, deve-se levar em conta que os quatro regimes, apresentados na Tabela 1, são caracterizados pelo MR-STVEC através da seguinte dinâmica:

- Regime 1: desvio negativo ao equilíbrio de longo prazo, ou seja, $\left(\sum_{h=1}^{h=h_{1}} \Delta \log P T F_{t-h+1}\right)_{t-d} \ll 0 \mathrm{e}\left(\sum_{h=1}^{h=h_{2}} \Delta \log P T F D_{t-h+1}\right)_{t-e} \ll 0$;

- Regime 2: retorno ao equilíbrio de longo prazo depois de desvio positivo da PTF, ou seja, e $\left(\sum_{h=1}^{h=h_{1}} \Delta \log P T F_{t-h+1}\right)_{t-d} \gg 0 \mathrm{e}\left(\sum_{h=1}^{h=h_{2}} \Delta \log P T F D_{t-h+1}\right)_{t-e} \ll 0$;

- Regime 3: retorno ao equilíbrio de longo prazo depois de desvio negativo da PTF, ou seja, $\left(\sum_{h=1}^{h=h_{1}} \Delta \log P T F_{t-h+1}\right)_{t-d} \ll 0 \mathrm{e}\left(\sum_{h=1}^{h=h_{2}} \Delta \log P T F D_{t-h+1}\right)_{t-e} \gg 0 ;$

- Regime 4: desvio positivo ao equilíbrio de longo prazo, ou seja, $\left(\sum_{h=1}^{h=h_{1}} \Delta \log P T F_{t-h+1}\right)_{t-d} \gg 0 \mathrm{e}\left(\sum_{h=1}^{h=h_{2}} \Delta \log \text { PTFD }_{t-h+1}\right)_{t-e} \gg 0$.

Feitas estas considerações, a partir dos resultados da Tabela 4, nota-se que o modelo $V E C$ linear estimado indica que, exceto no caso alemão, somente os coeficientes de ajustamento da equação da $P T F$ são estatisticamente significantes a níveis convencionais. Como salientado previamente, para países como os Estados Unidos e Japão, tal resultado não parece ser plausível dado que, ao longo de quase toda a segunda metade do século XX, choques positivos de produtividade, oriundos de outros fatores não associados ao setor externo, são restringidos pelas exportações.

Por outro lado, os resultados do MR-STVEC apresentam outra perspectiva. Para todos os casos, esse mecanismo de ajustamento vem ocorrer no regime 3, quando a PTF dessas economias encontra-se em fase de baixo crescimento, seguido de movimento de recuperação da PTFD. Após o sistema sofrer choques negativos de produtividade, há uma dinâmica de reversão, significando a existência de mecanismo de sustentação da PTF orientado pelas exportações.

O MR-STVEC estimado para Japão e Alemanha evidenciam que, por meio das exportações, o ajustamento de curto prazo vem ocorrer no regime 2. Por conseguinte, quando o log da PTF encontra-se em movimento de ascensão, seguido de queda da PTFD, EX é a variável que responde positivamente. Logo, choques que promovam maior nível de produtividade, seja ele oriundo do setor não exportador, serão traduzidos em aumento do nível de participação das exportações. Interessante observar que para os Estados Unidos não há evidência de qualquer tipo de resposta do setor exportador ao aumento de produtividade da economia, uma vez que o coeficiente de ajustamento no regime 2 não é estatisticamente significante a níveis convencionais. O que, portanto, leva ao questionamento sobre o quão sensível é o setor exportador aos choques positivos de produtividade.

O Canadá e a Alemanha são os países que destoam das outras duas economias pelo fato de possuírem coeficiente de ajustamento no regime 2 da equação do $\log$ da $P T F_{t}$ estatisticamente diferente de zero. Dessa forma, logo após a ocorrência de choques positivos de produtividade há trajetória de retorno ao patamar pré-choque. O que chama atenção, portanto, é que o nível de participação das exportações canadenses restringe possíveis choques positivos de produtividade. Esse aspecto pode ser explicado pelas características desse 
Tabela 4: Resultados da Estimação do MR-STVEC

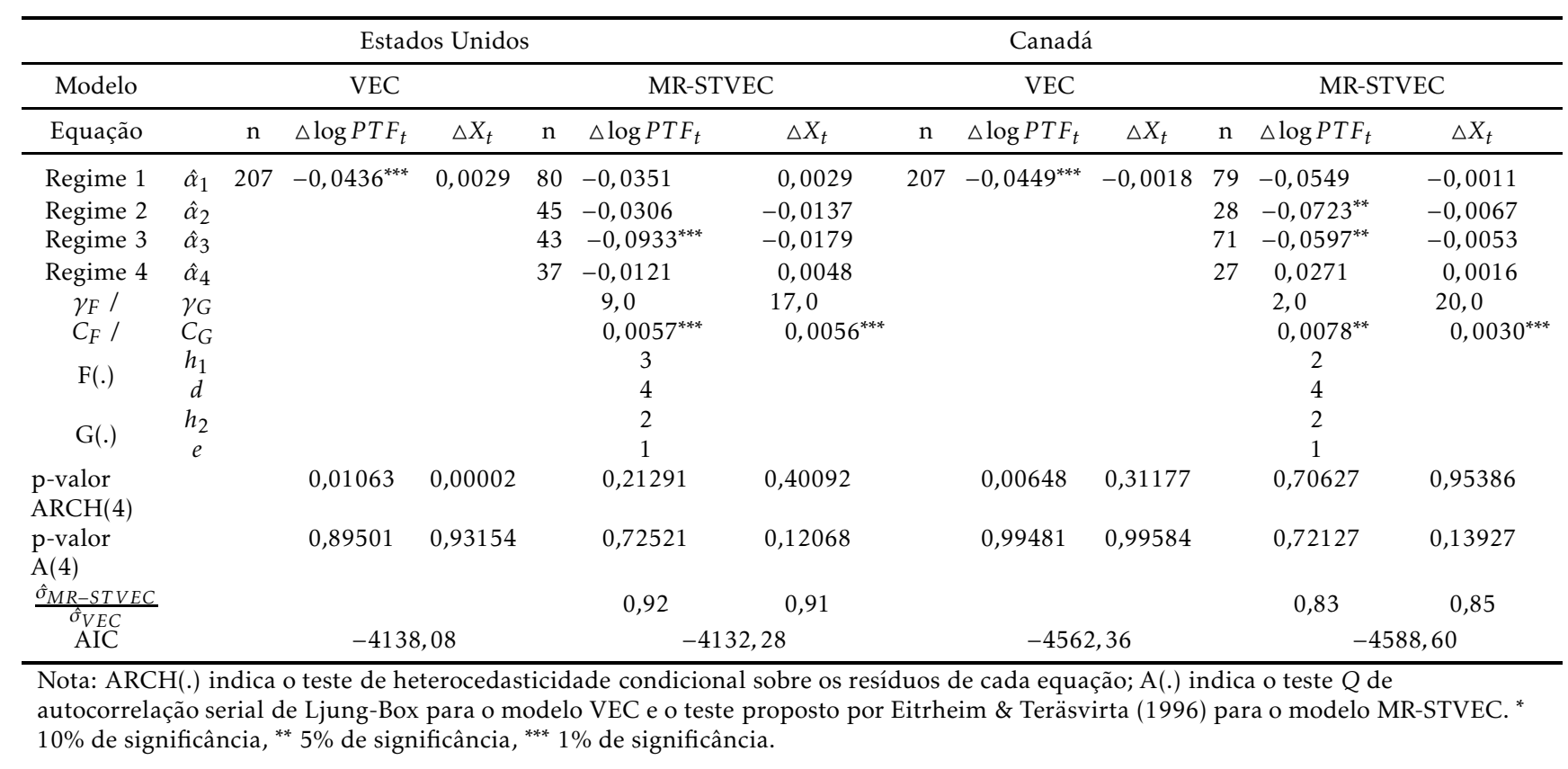


Tabela 4: Resultados da Estimação do MR-STVEC (continuação)

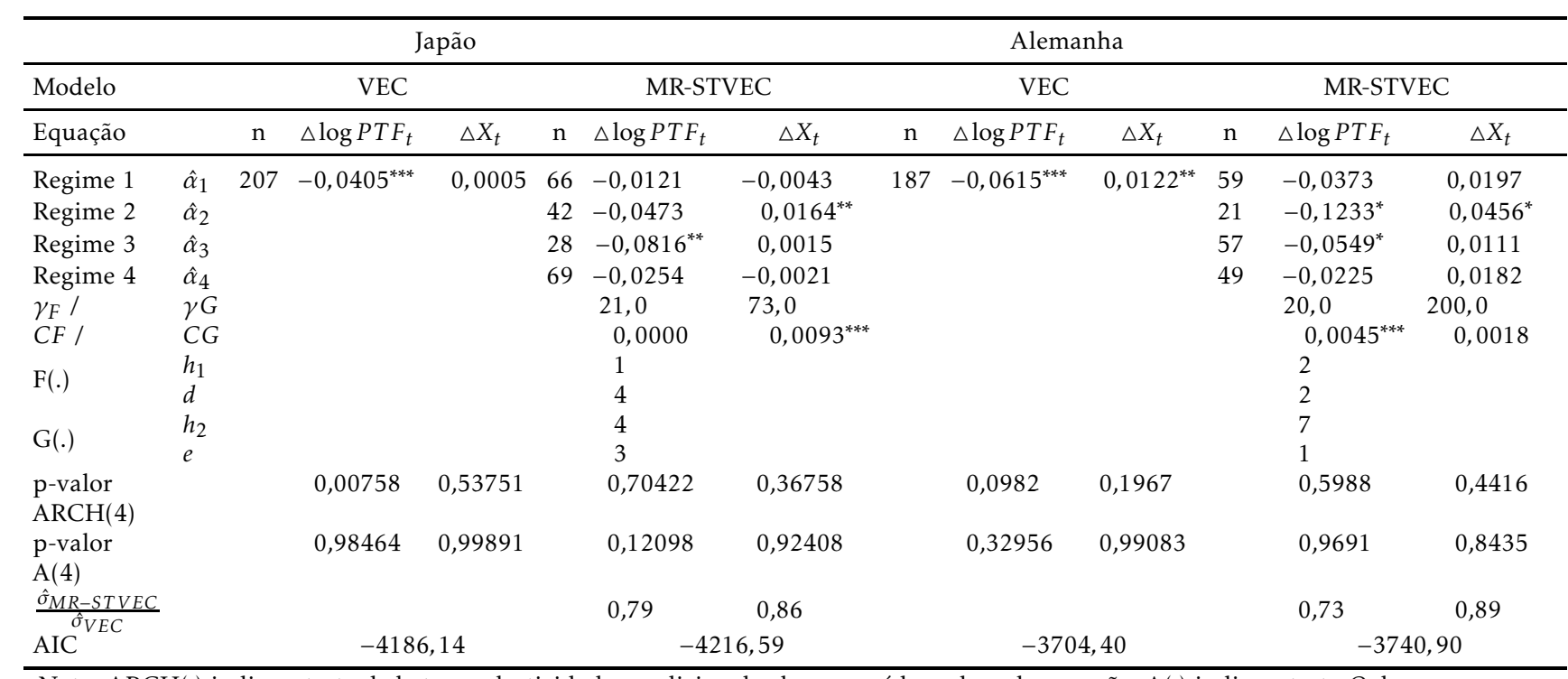

Nota: ARCH(.) indica o teste de heterocedasticidade condicional sobre os resíduos de cada equação; $\mathrm{A}($.$) indica o teste Q$ de

autocorrelação serial de Ljung-Box para o modelo VEC e o teste proposto por Eitrheim \& Teräsvirta (1996) para o modelo MR-STVEC. *

$10 \%$ de significância, ${ }^{* *} 5 \%$ de significância, ${ }^{* * *} 1 \%$ de significância. 
país que possui mercado doméstico relativamente pequeno e alta participação das exportações no PIB. Já no caso alemão, para o mesmo regime 2, as exportações também são responsáveis pelo ajustamento de curto prazo com a seguinte característica: o coeficiente $\hat{\alpha}_{21}$ (equação da $P T F_{t}$ ) superior a $\hat{\alpha}_{22}$ (equação de $E X_{t}$ ). Portanto, choques positivos sobre a PTF fazem com que o mecanismo de restrição seja parcial, tal como, há contrapartida positiva na participação de suas exportações no total exportado mundialmente.

Para melhor compreensão a respeito do processo de ajustamento de curto prazo nos modelos MR-STVEC, as Figuras 2 a 5, a seguir, apresentam a evolução das funções de transição conjuntamente com a série do log da $P T F_{t}$.

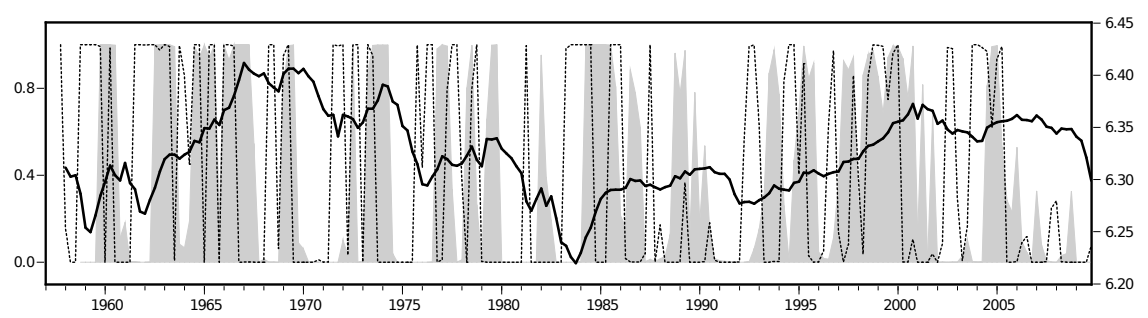

Figura 2: Evolução da função de transição $F($.$) (área), G($.$) (linha tracejada) e$ do $\log$ da $P T F_{t-4}$ (linha cheia) — Estados Unidos

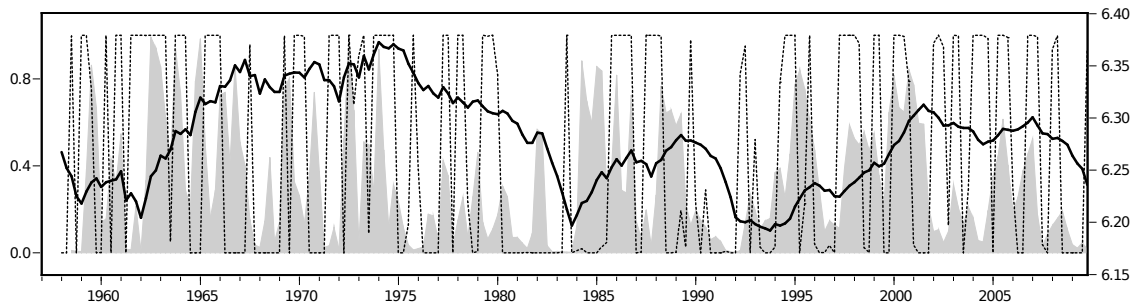

Figura 3: Evolução da função de transição $F($.$) (área), G($.$) (linha tracejada) e$ do $\log$ da $P T F_{t-4}$ (linha cheia) — Canadá

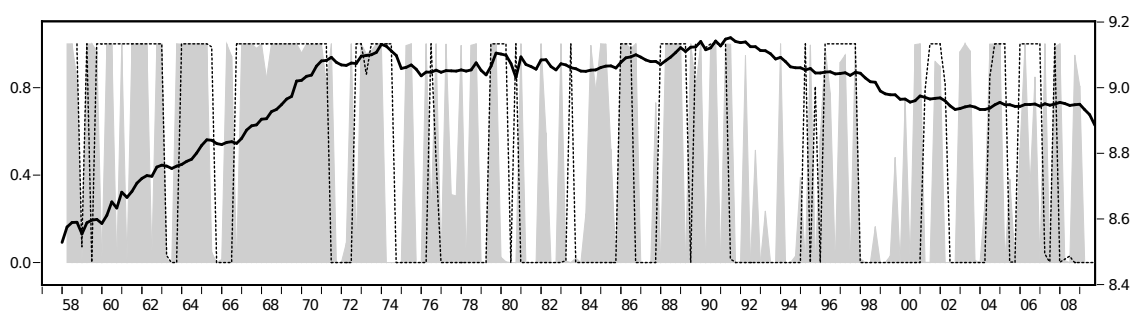

Figura 4: Evolução da função de transição $F($.$) (área), G($ (.) (linha tracejada) e do $\log$ da $P T F_{t-4}$ (linha cheia) — Japão

São perceptíveis os efeitos das exportações nos momentos em que a produtividade total dos fatores dessas economias encontra-se em trajetória de queda, observado quando $F($.) e $G($.) são inferiores a 0,5 nível em que ocorre a mudança de regime. Nos extremos das funções, essa dinâmica refere-se ao regime 1. De maneira geral, há reversão da queda do $\log$ da $P T F_{t}$ nos momentos 


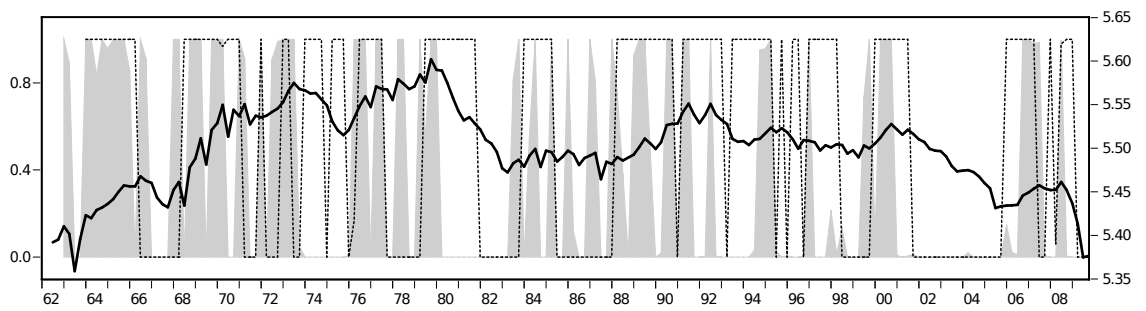

Figura 5: Evolução da função de transição $F($.$) (área), G($.$) (linha tracejada) e$ do log da $P T F_{t-2}$ (linha cheia) — Alemanha

em que $F($.$) é inferior a 0,5$ e $G($.$) superior a 0,5$ (regime 3 ). No caso do Canadá e Alemanha, verifica-se a situação inversa, uma restrição no crescimento do $\log$ da $P T F_{t}$ quando $F($.$) é superior a 0,5$ e $G($.$) inferior a 0,5$ (regime 2).

$\mathrm{O}$ processo de ajustamento por meio das exportações só ocorre para o modelo MR-STVEC do Japão e Alemanha. Para salvar espaço, nas Figuras 6 e 7 serão destacados somente a evolução do $E X_{t}$, conjuntamente com as funções de transição para esse dois países.

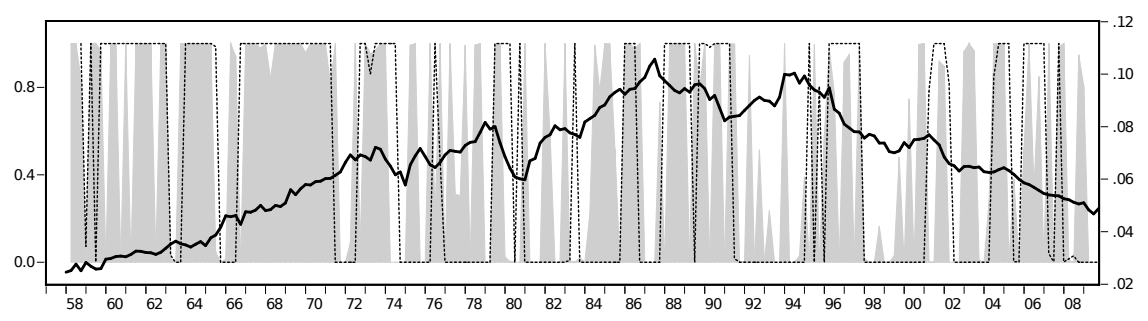

Figura 6: Evolução da função de transição $F($.$) (área), G($.$) (linha tracejada) e$ do índice $E X_{t-4}$ (linha cheia) - Japão

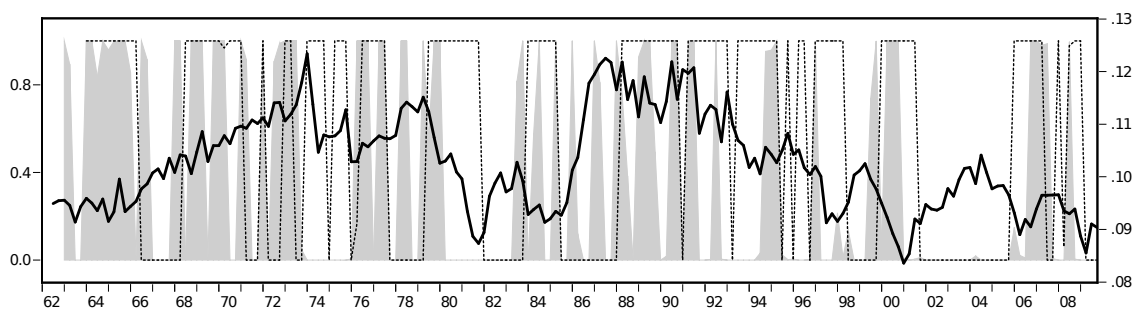

Figura 7: Evolução da função de transição $F($.$) (área), G($.$) (linha tracejada) e$ do índice $E X_{t-2}$ (linha cheia) - Alemanha

Observa-se que nesses casos, de maneira geral, há movimento de ascensão de $E X_{t}$ quando $F($.$) é superior a 0.5$ e $G($.) inferior a 0.5 (regime 2). Logo, como previsto no modelo estimado, a participação das exportações responde positivamente ao aumento da produtividade do país.

\section{Conclusão}

Este artigo constatou a existência de relação de longo prazo, nos últimos 50 anos, entre produtividade total dos fatores e exportações, para quatro países 
de renda per capita elevada, mas com distintos graus de participação do comércio internacional no PIB - Estados Unidos, Canadá, Japão e Alemanha. Diferentemente de grande parte da literatura econômica, optou-se por avaliar as exportações através de sua participação no valor total exportado pelo mundo. A partir do teste de especificação proposto, composto por dois procedimentos, verificou-se que a dinâmica de ajustamento pode ser caracterizada por meio do modelo MR-STVEC. Essa última especificação tem a vantagem sobre o modelo linear por tratar isoladamente os movimentos de desequilíbrio, seja dos desvios associados à incidência de choques negativos e positivos de produtividade, tal como dos retornos à trajetória de longo prazo.

No caso dos Estados Unidos, uma economia com baixa participação do setor exportador no PIB, o modelo linear indica que, independente do tipo de choque, a PTF sempre ajusta. Logo, as exportações agem de modo a restringir potenciais choques positivos de produtividade, o que é pouco factível para uma economia com mercado doméstico amplo. Por outro lado, o modelo não linear evidencia um processo condicionado de orientação da produtividade via exportações. Ou seja, a PTF ajusta somente quando há choques negativos de produtividade. Já o caso canadense também inclui dinâmica de ajustamento via PTF quando há choques positivos. Portanto, as exportações agem tanto de modo a sustentar a produtividade tal como restringi-la, com diferenças somente no que tange à velocidade do ajuste. Esse resultado é compatível para uma economia pequena como o Canadá que possui maior influência do setor exportador na economia.

O sentido de causalidade reversa que se processa da PTF para as exportações vem a ocorrer somente para Japão e Alemanha. O Japão, semelhantemente aos Estados Unidos, é uma das maiores economias do mundo, possui baixa participação das exportações em seu produto e tal como evidenciado pelo modelo MR-STVEC estimado, a orientação da PTF via exportações só ocorre quando há choques negativos de produtividade. Por outro lado, choques positivos de produtividade tem efeito sobre as exportações, de modo a elevar sua participação no total exportado no mundo.

O modelo estimado para Alemanha, tal como para os demais países, possui o mecanismo de sustentação da produtividade quando há choques negativos. Entretanto, com a incidência de choques positivos, tanto a PTF como as exportações ajustam, sendo que a velocidade de ajustamento da primeira é superior. Essa dinâmica de causalidade pode ser explicada, pois tal como Japão e Estados Unidos, a Alemanha é uma das maiores economias do mundo. Contudo, possui participação maior do setor exportador no produto, similar ao caso canadense. Observamos, portanto, que esse país não possui mecanismo exclusivo de ajustamento da PTF orientado pelas exportações. Inovações no setor que não atua no comércio internacional podem também influenciar o setor exportador, promovendo padrão de causalidade reversa.

\section{Agradecimentos}

Os autores agradecem os comentários e sugestões dos pareceristas da Revista Economia Aplicada, e dos professores Rodrigo de Losso da Silveira Bueno, Carlos Eduardo Soares Gonçalves, Sérgio Kannebley Júnior e Jaylson Jair da Silveira. 


\section{Referências Bibliográficas}

Anderson, H. M. \& Teräsvirta, T. (1992), 'Characterizing nonlinearities in business cycles using smooth transition autoregressive models', Journal of Applied Econometrics 7(S1), S119-S136.

Aw, B. Y., Chen, X. \& Roberts, M. J. (1997), 'Firm-level evidence on productivity diferentials, turnover, and exports in taiwanese manufacturing', NBER Working Paper n. 6235.

Awokuse, T. O. (2007), 'Causality between exports, imports, and economic growth: Evidence from transition economies', Economics Letters 94(3), 389395.

Balassa, B. (1978), 'Exports and economic growth: Further evidence', Journal of Development Economics 5(2), 181-189.

Bernard, A. B. \& Jensen, J. B. (1999), 'Exporting and productivity', National Bureau of Economic Research. Working Paper n. 7135.

Bernard, A. B., Jensen, J. B., Redding, S. J. \& Schott, P. K. (2007), 'Firms in international trade', The Journal of Economic Perspectives 21(3), 105-130.

Bhagwati, J. N. (1998), Protectionism, 1 edn, MIT Press.

Bruton, H. J. (1989), Import substitution, in H. CHENERY \& T. SRINIVASAN, eds, 'Handbook of Development Economics', Vol. 2, Oxford:North-Holland.

Clerides, S., Lach, S. \& Tybout, J. (1998), 'Is learning by exporting important? micro-dynamic evidence from colombia, mexico, and morocco', Quarterly Journal of Economics 113(3), 903-947.

Coe, D. \& Helpman, E. (1995), 'International r\&d spillovers', European Economic Review 39(5), 859-887.

Cooley, T. \& Prescott, E. (1995), Economic growth and business cycles, in T. COOLEY \& E. PRESCOTT, eds, 'Frontiers of Business Cycle Research', Vol. 1, Princeton University Press.

De Loecker, J. (2007), 'Do exports generate higher productivity? evidence from slovenia', Journal of International Economics 73(1), 69-98.

Dijik, D. V. \& Franses, P. H. (1999), 'Modeling multiple regime in the business cycle', Macroeconomic Dynamics 3(3), 311-340.

Dijik, D. V., Teräsvirta, T. \& Franses, P. H. (2002), 'Smooth transition autoregressive models - a survey of recent developments', Econometric Reviews $21(1), 1-47$.

Edwards, S. (1993), 'Openness, trade liberalization, and growth in developing countries', Journal of Economic Literature 31(3), 1358-1393.

Edwards, S. (1998), 'Openness, productivity and growth: what do we really know?', Economic Journal 108(447), 383-398.

Eitrheim, O. \& Teräsvirta, T. (1996), 'Testing the adequacy of smooth transition autoregressive models', Journal of Econometrics 74(1), 59-75. 
Esfahani, H. S. (1991), 'Exports, imports, and economic growth in semiindustrialized countries', Journal of Development Economics 35(1), 93-116.

Feder, G. (1982), 'On exports and economic growth', Journal of Development Economics 12(1), 59-73.

Giles, J. A. \& Williams, C. L. (2000a), 'Export-led growth: a survey of the empirical literature and some non-causality results. part 1', Journal of International Trade E Economic Development 9(4), 61-337.

Giles, J. A. \& Williams, C. L. (2000b), 'Export-led growth: a survey of the empirical literature and some non-causality results. part 2', Journal of International Trade E Economic Development 9(4), 445-470.

Granger, C. W. J. \& Teräsvirta, T. (1993), Modeling Nonlinear Economic Relationships, 1 edn, New York: Oxford University Press.

Greenaway, D. \& Sapsford, D. (1994), 'Exports, growth, and liberalization: An evaluation', Journal of Policy Modeling 16(2), 165-186.

Grossman, G. M. \& Helpman, E. (1991), Innovation and Growth in the Global Economy, 1 edn, Cambridge: MIT Press.

Hall, R. E. \& Jones, C. I. (1999), 'Why do some countries produce so much more output per worker than others?', The Quarterly Journal of Economics 114(1), 83-116.

Henriques, I. \& Sadorsky, P. (1996), 'Export-led growth or growth-driven exports? the canadian case', The Canadian Journal of Economics 29(3), 540-555.

Issler, J. V., Pessoa, S. A. \& Ferreira, P. C. (2004), 'Testing production functions used in empirical growth studies', Economic Letters 83(1), 29-35.

Johansen, S. (1988), 'Statistical analysis of cointegration vectors', Journal of Economics Dynamics and Control 12(2-3), 231-365.

Johansen, S. (1991), 'Estimation and hypothesis testing of cointegration vectors in gaussian vector autoregressive models', Econometrica 59(6), 15511580 .

Krueger, A. (1980), 'Trade policy as an input development', American Economic Review 70(2), 288-292.

Krugman, P. (1984), Import protection as export promotion, in H. Kierzkowski, ed., 'Monopolistic Competition in International Trade', Vol. 1, Oxford University Press.

Kunst, R. M. \& Marin, D. (1989), 'On exports and productivity: A causal analysis', The Review of Economics and Statistics 71(4), 699-703.

Lundbergh, S., Dijik, D. V. \& Teräsvirta, T. (2003), 'Time-varying smooth transition autoregressive models', Journal of Business E Economic Statistic 21(1), 104-121.

Mackinnon, J. G., Alfred, A. H. \& Michelis, L. (1999), 'Numerical distribution functions of likelihood ratio tests for cointegration', Journal of Applied Econometrics 14(5), 563-577. 
Marin, D. (1992), 'Is the export-led growth hypothesis valid for industrialized countries?', The Review of Economics and Statistics 74(4), 678-688.

Rodrik, D. (1995), 'Trade strategy, investment and export: Another look at east asia', National Bureau of Economic Researcher Working Paper 5339.

Rothman, P., Dijik, V. D. \& Franses, P. H. (2001), 'Multivariate star analysis of money-output relationship', Macroeconomics Dynamics 5(4), 506-532.

Teräsvirta, T. (1994), 'Specification, estimation, and evaluation of smooth transition autorregressive models', Journal of the American Statistical Association 89(425), 208-218.

Vamvakidis, A. (2002), 'How robust is the growth-openness connection? a historical evidence', Journal of Economic Growth 7(1), 57-80.

$\mathrm{Xu}, \mathrm{Z}$. (1996), 'On the causality between export growth and gdp growth: An empirical reinvestigation', Review of International Economics 4(2), 172-184.

Yamada, H. (1988), 'A note on the causality between export and productivity: an empirical re-examination', Economics Letters 61(1), 111-114.

\section{Apêndice A}

Considerando o modelo em (10), o cálculo de $\nabla H\left(z_{t-1}, \Delta x_{t-j}, s_{F_{t-d}} ; \hat{\Psi}\right)=\frac{\partial H\left(z_{t-1}, \Delta x_{t-j}, s_{F_{t-d}} ; \hat{\Psi}\right)}{\partial \hat{\Psi}}$ é dado por:

$$
\begin{gathered}
\frac{\partial H}{\partial \hat{\tilde{\mu}}_{1}}=1 \\
\frac{\partial H}{\partial \hat{\tilde{\alpha}}_{1}}=z_{t-1} \\
\frac{\partial H}{\partial \hat{\tilde{\Phi}}_{1, j}}=\Delta x_{t-j} \\
\frac{\partial H}{\partial \hat{\tilde{\mu}}_{2}}=F\left(s_{F_{t-d}} ; \hat{\gamma}_{F}, \hat{c}_{F}\right) \\
\frac{\partial H}{\partial \hat{\tilde{\alpha}}_{2}}=z_{t-1} F\left(s_{F_{t-d}} ; \hat{\gamma}_{F}, \hat{c}_{F}\right) \\
\frac{\partial H}{\partial \hat{\tilde{\Phi}}_{2, j}}=\Delta x_{t-j} F\left(s_{F_{t-d}} ; \hat{\gamma}_{F}, \hat{c}_{F}\right) \\
\frac{\partial H}{\partial \hat{\gamma}_{F}}=\left(1+\exp \left\{-\hat{\gamma}_{F}\left(s_{F_{t-d}}-\hat{c}_{F}\right)\right\}\right)^{-2} \exp \left\{-\hat{\gamma}_{F}\left(s_{F_{t-d}}-\hat{c}_{F}\right)\right\} * \\
\left(s_{F_{t-d}}-\hat{c}_{F}\right)\left(\hat{\tilde{\mu}}_{2}+\hat{\tilde{\alpha}}_{2} z_{t-1}+\sum_{j=1}^{p} \hat{\tilde{\Phi}}_{2, j} \Delta x_{t-j}\right) \\
\frac{\partial H}{\partial \hat{c}_{F}}=\hat{\gamma}_{F}\left(1+\exp \left\{-\hat{\gamma}_{F}\left(s_{F_{t-d}}-\hat{c}_{F}\right)\right\}\right)^{-2} \exp \left\{-\hat{\gamma}_{F}\left(s_{F_{t-d}}-\hat{c}_{F}\right)\right\} * \\
\left(\hat{\hat{\mu}}_{2}+\hat{\alpha}_{2} z_{t-1}+\sum_{j=1}^{p} \hat{\tilde{\Phi}}_{2, j} \Delta x_{t-j}\right)
\end{gathered}
$$




\section{Apêndice B}

Tabela B.1: Teste Traço de Razão de Verossimilhança

\begin{tabular}{lcccc}
\hline País & $\mathrm{n}$ & $\mathrm{p}$ & Estatística Traço & Vetor de Cointegração \\
\hline Estados Unidos & 212 & 4 & $16,3^{* *}$ & {$[1-1,46-6,14]$} \\
Canadá & 212 & 4 & $15,1^{*}$ & {$[1-7,56-5,96]$} \\
Japão & 212 & 4 & $20,7^{* *}$ & {$[1-3,13-8,79]$} \\
Alemanha & 192 & 4 & $20,5^{* *}$ & {$[1-5,73-4,91]$} \\
\hline $\begin{array}{l}\text { Notas: Os valores críticos teste traço são obtidos de Mackinnon et al. }(1999) .^{*} \\
\text { significante a 10\%, }\end{array}$ \\
para Estados Unidos e Canadícante a 5\% e e e $^{* * *}$ significante a 1\%. Os modelos estimados constante no vetor de correção de erros.
\end{tabular}

